\title{
Fibrosis endomiocárdica crónica, reporte de caso en área no endémica
}

\author{
Marco Tobar ${ }^{a, *}$, Roberto Basante ${ }^{a}$, María Guerrero $^{a}$ \\ 1. Médico residente de medicina interna, Hospital Regional Licenciado Adolfo López Mateos, Universidad \\ Nacional Autónoma de México. Ciudad de México.
}

APOYO FINANCIERO: Ios autores declaran que no recibieron ayuda económica de terceras personas

para la realización de este artículo.

\section{Chronic endomyocardial fibrosis, case report in a non-endemic area}

Endomyocardial fibrosis is a known and prevalent cause of restrictive cardiomyopathy in countries of Equatorial Africa, but very rare elsewhere. Although the diagnosis in endemic countries is established by the presence of certain echocardiographic criteria, the gold standard is the endomyocardial biopsy, which shows fibrous tissue in the endocardium. An acute and subacute phase can be distinguished, with associated mortality due to com- plications of heart failure. In contrast, the chronic phase presents less aggressive behavior, with progression of symptoms. In this phase, surgical treatment may improve survival. We present the case of a 44-year-old patient with good clinical control under pharmacological treatment after 14 months of follow-up.

Keywords: Endomyocardial fibrosis, restrictive cardiomyopathy, heart failure.

\section{Correspondencia:}

Dr. Marco Adolfo Tobar Marcillo

Avenida Gabriel Mancera 1258, Colonia del Valle, Delegación

Benito Juárez, Ciudad de México.

marcotobar1@hotmail.com 


\section{Introducción:}

La miocardiopatía restrictiva es una entidad desencadenada por un amplio grupo de patologías de diferente etiología que comparte manifestaciones clínicas comunes ${ }^{1}$, que en más del $90 \%$ de pacientes es falla cardíaca que, generalmente, es el motivo de internación. El síntoma más frecuente es la disnea, pero por el compromiso derecho se suele acompañar de edemas, hepatomegalia y ascitis.

Se presenta el caso clínico de una mujer de 44 años que se interna en el servicio de cardiología de una institución de tercer nivel en la Ciudad de México y luego de protocolo diagnóstico por medio de biopsia se determina como etiología una fibrosis endomiocárdica.

\section{Presentación del caso}

Paciente femenina de 44 años de edad, procedente, y originaria, de la Ciudad de México, sin antecedentes de enfermedades crónico-degenerativas de importancia. Sin antecedentes quirúrgicos, alérgicos y transfusionales, tabaquismo ni alcoholismo. Sus antecedentes ginecológicos eran G1P1V1A0 con ciclos regulares de 30 x 4 . Es secretaria, con nivel socioeconómico medio. Consulta por cuadro clínico de 6 meses de evolución de disnea progresiva, ortopnea y edema de miembros inferiores, cuadro exacerbado en las últimas dos semanas con disnea de reposo, palpitaciones y dolor en hipocondrio derecho. A su ingreso presenta fibrilación auricular con respuesta ventricular rápida 118 latidos por minuto, hipoxemia con SAT O2 84\% y PaO2 54mmHg, ingurgitación yugular de $5 \mathrm{~cm}$, hepatomegalia, ascitis grado 2 , edema de miembros inferiores grado 2 y acropaquias. A la auscultación cardiopulmonar tenía un soplo grado IV/IV pansistólico continuo más intenso en foco mitral y tricuspídeo y estertores en bases pulmonares. Se hospitaliza con diagnóstico de insuficiencia cardíaca descompensada y se inicia estudio con un ecocardiograma realizado en fibrilación auricular. Se demuestra un crecimiento biauricular severo, aurícula izquierda con volumen de $81 \mathrm{ml}$ y aurícula derecha de $76.2 \mathrm{ml}$, válvula mitral con área $2.2 \mathrm{~cm} 2$, con ligero engrosamiento de valva antero medial sin otras lesiones aparentes; flujo mitral por doppler pulsado con onda E $83 \mathrm{~cm} /$ segundo, doppler tisular mitral con onda $\mathrm{E}^{\prime}$ en anillo mitral lateral de $4.8 \mathrm{~cm} /$ segundo y en anillo mitral medial $4 \mathrm{~cm} /$ segundo, con relación E/E' calculada con relación al anillo mitral lateral de 17.29 y al anillo mitral medial 20.75, con claro patrón restrictivo.

El diámetro de ventrículo izquierdo conservado, con fracción de eyección calculada por Simpson de $68 \%$, espesor de septum $13 \mathrm{~mm}$ y pared libre de $11 \mathrm{~mm}$ con presencia de placas endocárdicas en pared septal y lateral de $3 \mathrm{~mm}$ de espesor; válvula aórtica trivalva, con área valvular de $2.6 \mathrm{~cm} 2$, gradiente medio de $13 \mathrm{mmHg}$, con ligera calcificación, sin lesiones vegetantes. En la valoración de cavidades derechas se observa diámetro de anillo tricuspídeo de $2.8 \mathrm{~cm} 2$, con jet de regurgitación de 2.3 $\mathrm{m} /$ segundo, cálculo de presión sistólica de arteria pulmonar estimado de $29 \mathrm{mmHg}$, desplazamiento sistólico del anillo tricúspideo de $23.3 \mathrm{~mm}$, onda $\mathrm{S}^{\prime}$ del ventrículo derecho en doppler tisular pulsado de $21.8 \mathrm{~cm}$ y con obliteración en su ápex. En espacio pericárdico se demuestra derrame ligero cuantificado en 110ml. (Figura 1).

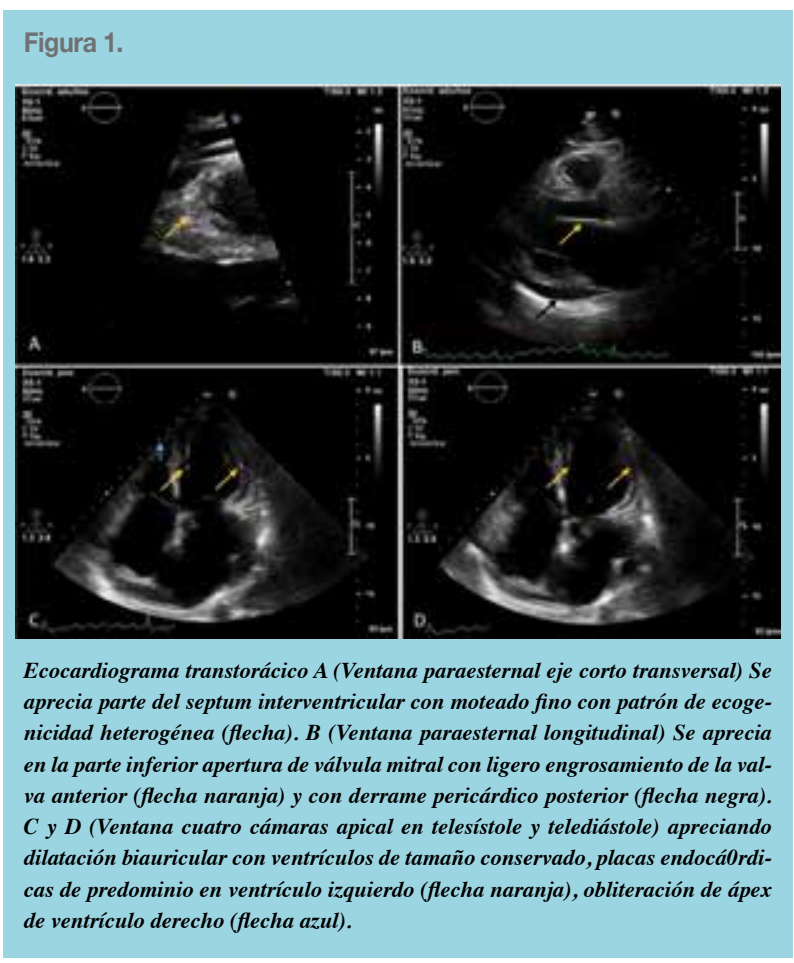

La función renal estaba conservada, electrolitos en equilibrio, la hemoglobina era $13.0 \mathrm{gr} / \mathrm{dl}$, el volumen corpuscular medio de 90.1 fl, HCM 30 pg, leucocitos de 9280/ $\mu \mathrm{L}$, neutrófilos de $7460 / \mu \mathrm{L}$, eosinófilos de $150 / \mu \mathrm{L}$, plaquetas $172.000 / \mu \mathrm{L}$.

Se diagnostica miocardiopatía restrictiva y se inicia estudio etiológico descartando, en orden de frecuencia, esclerosis sistémica con anticuerpos anti-Scl $70 \mathrm{y}$ anti centrómero negativos, sin compromiso de otros órganos; enfermedades infiltrativas tipo amiloidosis con tejido celular subcutáneo negativo para coloración de rojo Congo, ausencia de compromiso renal y hepático; se descartó por antecedentes enfermedades de depósito lisosomal Hur- 
ler y Fabry, y perfil TORCH negativo. Ante ausencia de diagnóstico se realiza un estudio de hemodinamia que reporta coronarias sin lesiones, ventriculografía derecha e izquierda con signo de raíz cuadrada positivo y datos indicativos de fibrosis severa (Figura 2).

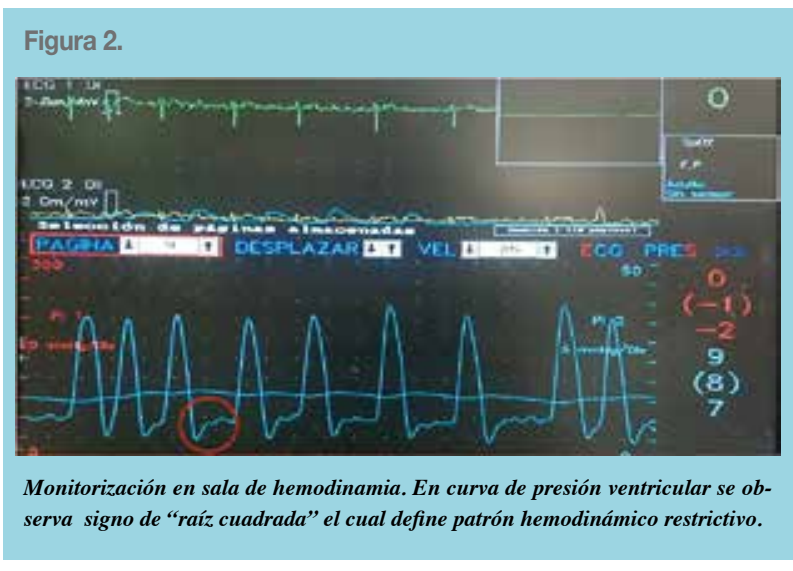

Se realizó biopsia del ventrículo derecho a nivel del septum interventricular y el estudio histopatológico bajo tinción tricrómica de Masson y hematoxilina eosina fue compatible con fibrosis endomiocárdica (Figura 3).

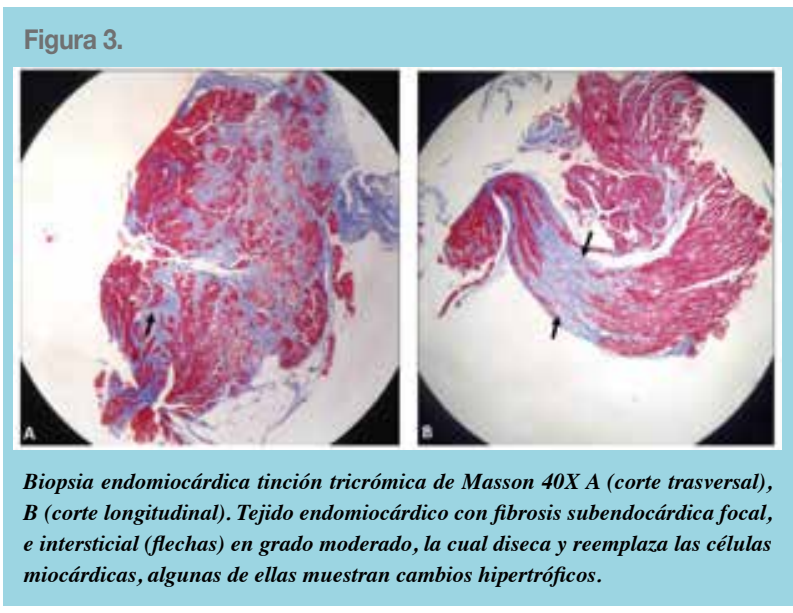

Se inició tratamiento farmacológico de falla cardíaca aguda con diuréticos, inhibidores de la enzima convertidora de angiotensina (IECA's) y oxigenoterapia con rápida mejoría. Se dio de alta con enalapril, metoprolol succinato, furosemida, espironolactona y anticoagulación con rivaroxabán y se encuentra en seguimiento ambulatorio durante 14 meses. Su clase funcional es NYHA ${ }^{2}$, en fibrilación auricular permanente con adecuado control de respuesta ventricular. Se ha diferido el tratamiento qui- rúrgico, por no progresión de los síntomas con adecuada adherencia al tratamiento instaurado.

\section{Discusión:}

Las miocardiopatías restrictivas son afecciones caracterizadas por una restricción al llenado ventricular causada por tejido endocárdico, subendocárdico o miocárdico anormal que produce una alteración de la distensibilidad ventricular. Clásicamente se manifiesta como insuficiencia cardíaca con síntomas predominantes secundarios a falla de cavidades derechas ${ }^{1}$.

Es importante el correcto abordaje etiológico que permita la instauración rápida del tratamiento, a fin de mejorar el pronóstico en estos pacientes que, por lo general, son adultos jóvenes. Una de sus causas reconocidas es la enfermedad de Davies o fibrosis endomiocárdica, una enfermedad de etiología desconocida endémica en países tropicales principalmente en África ecuatorial. Históricamente, se relaciona con la endocarditis de Löeffler, actualmente diferenciadas porque la eosinofilia sérica y miocárdica no se presenta consistentemente en la fibrosis endomiocárdica, mientras que en la endocarditis de Löeffler es una condición imprescindible. Además, el curso clínico es más insidioso con progresión menos agresiva de la fibrosis ${ }^{2}$.

La fibrosis endomiocárdica es una enfermedad muy infrecuente en México encontrándose pocos casos reportados en la literatura. La mayor incidencia de esta patología se presenta en la región del África ecuatorial, en especial Uganda, sitio donde se describió por primera vez ${ }^{3}, \mathrm{Se}$ encuentra además de India y China, y en Sur América, Brasil y Colombia. Afecta apersonas jóvenes con una distribución bimodal a los 10 y 30 años, afectando ligeramente más a mujeres que a hombres ${ }^{4}$.

$\mathrm{Su}$ etiología es multifactorial interviniendo factores ambientales, dietéticos, como el bajo nivel de magnesio, toxicidad por cerio ${ }^{5}$ e infecciones previas, en especial malaria ${ }^{6}$, enfermedad de $\mathrm{Chagas}^{7}$, y otras relacionadas con elevación de eosinófilos en sangre. Hay presencia de anticuerpos contra proteínas cardíacas, sin lograr establecer una clara relación causal, en parte por la distribución geográfica $^{8}$. No se han encontrado datos que sugieran una predisposición genética, puesto que existen muchos reportes en migrantes que previamente habitaban áreas no endémicas.

En el año 2008, Mocumbi et $\mathrm{al}^{9}$, realizaron un estudio de campo en 214 familias de áreas rurales de Mozambique, estableciendo criterios diagnósticos de fibrosis endomiocárdica, en la que previamente el "gold standard" era la 


\begin{tabular}{|l|c|}
\hline TABLA 1 Criterios diagnósticos de fibrosis endomiocárdica (9) & Puntaje \\
\hline CRITERIOS MAYORES & \\
\hline Placas > 2 mm de espesor en el endomiocardio & 2 \\
\hline Parches menores 1 mm en el endomiocardio, afectando más de una pared del ventrículo & 3 \\
\hline Obliteración del ápex ventricular & 4 \\
\hline Trombo espontáneo o eco contraste sin presencia de disfunción ventricular & 4 \\
\hline Retracción del ápex del ventrículo derecho & 4 \\
\hline Disfunción de válvulas AV por adhesión del aparato subvalvular a la pared del ventrículo & \\
\hline *De acuerdo al grado de insuficiencia valvular se da el puntaje & $1-4^{\star}$ \\
\hline CRITERIOS MENORES & \\
\hline Parches delgados de endomiocardio localizados en una pared ventricular & 1 \\
\hline Patrón restrictivo a través de las válvulas auriculo ventriculares & 2 \\
\hline Apertura en diástole de la válvula pulmonar & 2 \\
\hline Engrosamiento difuso de la valva anterior mitral & 1 \\
\hline Crecimiento de aurícula con ventrículos normales & 2 \\
\hline Realce en la densidad de la banda moderadora o de las trabéculas & 1 \\
\hline En modo M: movimiento del septum interventricular y la pared posterior & 1 \\
\hline
\end{tabular}

biopsia endomiocárdica. En este caso, pese a cumplir criterios ecocardiográficos con un total de 13 puntos, que corresponde a una fibrosis moderada, se optó por hacer la biopsia, por ser una patología muy infrecuente en México (Tabla 1).

En la evolución clínica se distingue una fase aguda donde la característica predominante es la infiltración de eosinófilos y tejido inflamatorio en el endocardio. Se presenta clínicamente como una insuficiencia cardíaca aguda y en el ecocardiograma se observa un patrón de hiperrefringencia con infiltrados homogéneos en todo el espesor de la pared miocárdica. Por mecanismos desconocidos, algunos pacientes progresan a falla cardíaca severa y fallecen, y otros pacientes entran en fase subaguda, donde la enfermedad tiene progresión lenta, predominando sintomatología de falla cardíaca crónica con arritmias, especialmente, fibrilación auricular en el 30\% de los pacientes ${ }^{10}$. Además, hay trastornos de conducción con bloqueo aurícula ventricular. La biopsia muestra tejido fibróticas con escaso o nulo tejido inflamatorio ${ }^{11}$. También existe un grupo de pacientes que presenta regresión de síntomas sin presentar secuelas ${ }^{12}$.
El tratamiento consiste en tratar la falla cardíaca, donde la piedra angular está dada por el uso de IECA's, beta-bloqueadores y diuréticos. En caso de presentar fibrilación auricular la anticoagulación está indicada ${ }^{4}$. Existe consenso en que el tratamiento quirúrgico, que consiste en endocardiectomía con resección de material fibroso más valvulopatía o reemplazo valvular mitral y tricuspídeo, incrementa la supervivencia ${ }^{13,14}$. En este caso se dio tratamiento médico con buena evolución clínica, sin progresión de la enfermedad en 14 meses de seguimiento.

\section{Conclusión:}

La fibrosis endomiocárdica es una causa conocida y prevalente de miocardiopatía restrictiva en países del África ecuatorial. En este caso, la enfermedad ocurrió en un área no endémica, donde es una condición muy rara. La enfermedad, pese a ser de curso menos agresivo que la endocarditis de Löeffler, tiene un mal pronóstico con alta mortalidad en fases agudas y subagudas, presentado un comportamiento progresivo de falla cardíaca en su fase crónica. En esta etapa la cirugía ha mostrado incrementar la sobrevida. 


\section{Referencias}

1. KUSHWAHA S, FALLON J, FUSTER V. Restrictive Cardiomyopathy. New England Journal of Medicine. 1997; 336: 267-276.

2. FREERS J, MASEMBE V, SCHMAUZ R, MAYANJA-KIZZA H. Endomyocardial fibrosis syndrome in Uganda. The Lancet. 2000; 355: 1994-1995.

3. DAVIES JNP. Endocardial fibrosis in Africans: a heart disease of obscure aetiology in Africans. East Afr Med J. 1948; $25: 10-16$.

4. GRIMALDI A, MOCUMBI A, FREERS J, LACHAUD M, MIRABEL M, FERREIRA B, et al. Tropical Endomyocardial Fibrosis. Circulation. 2016; 133: 2503-2515.

5. KUMARI KT, RAVIKUMAR A, KURUP PA. Accumulation of glycosaminoglycans associated with hypomagnesaemia in endomyocardial fibrosis in Kerala: possible involvement of dietary factors. Indian Heart J. 1997; 49: 49-51.

6. MOCUMBI AO, SONGANE M, SALOMÃO C, ULIBARRI R, FERREIRA MB, YACOUB MH. Lack of evidence of myocardial damage in children with Plasmodium falciparum severe and complicated malaria from an endemic area for endomyocardial fibrosis. J Trop Pediatr. 2011; 57: 312-314.

7. WAYENGERA M. Searching for new clues about the molecular cause of endomyocardial fibrosis by way of in silico proteomics and analytical chemistry. PLoS One. 2009; 4: e7420.
8. FRANCO-PAREDES C, ROUPHAEL N, MÉNDEZ J FOLCH E, RODRÍGUEZ-MORALES AJ, SANTOS JI, et al. Cardiac manifestations of parasitic infections part 3: pericardial and miscellaneous cardiopulmonary manifestations. Clin Cardiol. 2007; 30: 277-280.

9. MOCUMBI A, FERREIRA M, SIDI D, YACOUB M. A Population Study of Endomyocardial Fibrosis in a Rural Area of Mozambique. New England Journal of Medicine. 2008; 359: 43-49.

10. THARAKAN JA. Electrocardiogram in endomyocardial fibrosis. Indian acing Electrophysiol J. 2011; 11: 129-133.

11. HASSAN WM, FAWZY ME, AL HELALY S, HEGAZY H, MALIK S. Pitfalls in diagnosis and clinical, echocardiographic, and hemodynamic findings in endomyocardial fibrosis: a 25-year experience. Chest. 2005; 128: 3985-3992.

12. PARRY EH, ABRAHAMS DG. The natural history of endomyocardial fibrosis. Q J Med. 1965; 34: 383-408.

13. SCHNEIDER U, JENNI R, TURINA J, TURINA M, HESS OM. Long-term follow up of patients with endomyocardial fibrosis: effects of surgery. Heart. 1998;79:362-367.

14. UENISHI E, CELEMÍN G, ARIAS C, CARVAJAL M, ARIZA LA ROTTA C. Reporte de un caso de endomiocardiofibrosis en Colombia. Revista Colombiana de Cardiología, 2014; 21: 414-418. 